\title{
USO DE SUBSTÂNCIAS PSICOATIVAS ENTRE PROFISSIONAIS DA ENFERMAGEM DA ATENÇÃO BÁSICA E INSTITUIÇÃO HOSPITALAR
}

\section{USE OF PSYCHOACTIVE SUBSTANCES AMONG BASIC ATTENTION NURSING PROFESSIONALS AND HOSPITAL INSTITUTION}

\section{USO DE SUSTANCIAS PSICOACTIVAS ENTRE LOS PROFESIONALES DE ENFERMERÍA DE ATENCIÓN PRIMARIA Y LOS DE INSTITUCIONES HOSPITALARIAS}

Andressa Domingues Silva ${ }^{1}$, Emiliana Cristina Melo ${ }^{2}$, Júlia Trevisan Martins ${ }^{3}$, Camila Dálcol ${ }^{4}$, Edivaldo Cremer ${ }^{5}$, Alessandro Rolim Scholze ${ }^{6}$.

\section{RESUMO}

Objetivo: Comparar o uso de substâncias psicoativas em profissionais da enfermagem da atenção básica e de instituição hospitalar. Método: Estudo transversal, desenvolvido com profissionais de enfermagem, utilizando-se dois instrumentos: caracterização sociodemográfica e ocupacional e o Alcohol, Smoking and Substance Involvement Screening Test. Na análise dos dados, utilizou-se estatística descritiva e o teste qui-quadrado para verificar as associações. Resultados: O consumo de álcool e tabaco na vida e, nos três últimos meses, foi, proporcionalmente, maior entre profissionais da atenção básica. O uso de sedativos foi maior nas instituições hospitalares, sobretudo o uso na vida $(p=0,05)$. O forte desejo de álcool e sedativos esteve presente entre profissionais das instituições hospitalares e para o tabaco foi maior nos profissionais da atenção básica. Conclusão: Ao comparar, nota-se que houve uso distinto de drogas entre os profissionais das instituições hospitalares e os profissionais da atenção básica.

Descritores: Equipe de Enfermagem; Transtornos Relacionados ao Uso de Substâncias; Bebidas alcoólicas; Saúde do trabalhador; Saúde mental.

\section{ABSTRACT}

Objective: to compare the use of psychoactive substances in nursing professionals in primary care and hospital institutions. Method: a cross-sectional study developed with nursing professionals, using two instruments: sociodemographic and occupational characterization and the Alcohol, Smoking and Substance Involvement Screening Test. In the data analysis, descriptive statistics and the chi-square test were used to verify associations. Results: the consumption of alcohol and tobacco in life and in the last three months was proportionally higher among primary care professionals. The use of sedatives was greater in hospital institutions, especially the use in life $(p=0.05)$. The strong desire for alcohol and sedatives was observed among professionals in hospital institutions, whereas the need for tobacco was greater among professionals in primary care. Conclusion: Different drug uses were observed when comparing professionals from hospital institutions and professionals from primary care.

Descriptors: Nursing, Team; Substance-Related Disorders; Alcoholic Beverages; Occupational Health; Mental Health.

\section{RESUMEN}

Objectivo: comparar el uso de sustancias psicoactivas entre los profesionales de enfermería de la atención primaria y los de instituciones hospitalarias. Método: estudio transversal, desarrollado con profesionales de enfermería, utilizando dos instrumentos: caracterización sociodemográfica y ocupacional y el Alcohol, Smoking and Substance Involvement Screening Test. En el análisis de los datos se utilizo estadística descriptiva y el test de chi-cuadrado para verificar las asociaciones Resultados: el consumo de alcohol y tabaco a lo largo de la vida y en los últimos tres meses fue proporcionalmente mayor entre los profesionales de la atención primaria. El uso de sedantes fue mayor en las instituciones hospitalarias, especialmente el uso a lo largo de la vida $(p=0.05)$. El fuerte deseo de alcohol y sedantes estaba presente entre los profesionales de las instituciones hospitalarias y el consumo de tabaco fue mayor entre los profesionales de la atención primaria. Conclusión: al compararlos se observa que hubo un uso diferente de medicamentos entre los profesionales de instituciones hospitalarias y los profesionales de atención primaria.

Descriptores: Grupo de Enfermería; Trastornos Relacionados con Sustancias; Bebidas Alcohólicas; Salud Laboral; Salud Mental.

${ }^{1}$ Enfemeira, Universidade Estadual do Norte do Paraná. ${ }^{2}$ Doutora em enfermagem, Docente do curso de enfermagem da Universidade Estadual do Norte do Paraná. ${ }^{3}$ Doutora em enfermagem, Docente do curso de Pós-graduação em enfermagem da Universidade Estadual de Londrina. ${ }^{4}$ Mestra em enfermagem, Docente do curso de enfermagem da Universidade Estadual do Norte do Paraná. ${ }^{5}$ Doutor em ciências da saúde, Docente do curso de enfermagem da Universidade Estadual do Norte do Paraná. ${ }^{6}$ Mestre em enfermagem, Docente do curso de enfermagem da Universidade Estadual do Norte do Paraná.

Como citar este artigo:

Silva AD, Melo EC, Martins JT, et al. Uso de substâncias psicoativas entre profissionais da enfermagem da Atenção Básica e Instituição Hospitalar. Revista de Enfermagem do Centro Oeste Mineiro. 2020;10:e3737. [Access DOI: http://doi.org/10.19175/recom.v10i0.3737 


\section{INTRODUÇÃO}

O uso de Substâncias Psicoativas (SPA) é um grave problema de saúde pública, em todo o mundo, sendo este um fator que gera prejuízos no âmbito social e na saúde ${ }^{(1-2)}$. Dados mostram que a prevalência do uso de SPA (exceto o álcool) é de $5,2 \%$ na população mundial, que corresponde a 243 milhões de pessoas que consomem algum tipo de substância ${ }^{(3)}$. Na Europa, no ano de 2015, de cada três pessoas, ao menos uma relatou um episódio de consumo excessivo de álcool (consumo superior a cinco doses em uma única ocasião) nos últimos 30 dias, e, de cada cinco pessoas, uma possuía o hábito de fumar diariamente e $7 \%$ consumiram cannabis no último ano ${ }^{(2)}$.

O consumo das SPA, principalmente as lícitas, é um hábito recreativo e socialmente aceitável, utilizado com o objetivo de promover relaxamento, interação social e outros ${ }^{(4)}$. No entanto, quando esse hábito se torna abusivo, deixa $o$ indivíduo suscetível a consequências negativas para a sua saúde, entre elas: acidentes de trânsito, violência física e verbal, relação sexual desprotegida, consumo de outras substâncias ilícitas, prejuízos nos relacionamentos sociais e no ambiente ocupacional, bem como desencadeia uma maior susceptibilidade para 0 desenvolvimento de dependência química ${ }^{(4,5)}$.

O uso indiscriminado de SPA não apresenta uma diferenciação entre grupos populacionais, sendo este um hábito observado nos mais diferentes cenários populacionais, inclusive entre os profissionais da enfermagem ${ }^{(4,6-7)}$. Em relação à equipe de enfermagem, a prevalência do consumo de SPA tem variado entre $6 \%$ e $8 \%$, podendo ser ainda maior, quando analisado o uso abusivo de sedativos $20 \%{ }^{(3)}$.

Quando um trabalhador desenvolve o hábito de consumir alguma SPA, esse ato pode gerar complicações significativas no ambiente de trabalho, como redução da produtividade e percepção das atividades, maior susceptibilidade para os acidentes de trabalho, insatisfação com seu ambiente ocupacional e maiores índices de absenteísmo ${ }^{(3)}$.

A enfermagem encontra-se inserida em todos os níveis de atenção à saúde, entre eles, a atenção básica e média complexidade. Esses ambientes apresentam características similares como ambiente insalubre, baixa satisfação laboral, condições de trabalho precário, déficit nos relacionamentos interpessoais e multiprofissionais, aspectos estruturais e organizacionais, falta de participação e envolvimento nas decisões do serviço, portanto, todos esses fatores favorecem para o adoecimento mental e, muitas vezes, na busca por alternativas para aliviar as tensões relacionadas ao ambiente ocupacional recorrem ao consumo de SPA ${ }^{(8)}$.

Embora o uso de SPA entre profissionais da saúde já tenha sido objeto de pesquisas, ainda há a necessidade de estudos que comparem o uso dessas substâncias entre profissionais da atenção básica e de instituições hospitalares, visto que possuem processos de trabalho diferentes. Os achados poderão fornecer subsídios aos gestores e aos trabalhadores de enfermagem na execução de ações preventivas, de redução de danos e de tratamento de acordo com as diferentes necessidades. Assim, neste estudo, objetivou-se comparar o uso de substâncias psicoativas em profissionais da enfermagem da atenção básica e de instituição hospitalar.

\section{MÉTODO}

Estudo transversal, desenvolvido com profissionais da enfermagem (auxiliar de enfermagem, técnico de enfermagem e enfermeiros) de 13 Unidades Básicas de Saúde (UBS) e duas instituições hospitalares de média complexidade de quatro municípios de pequeno porte do interior do Estado do Paraná.

O critério de inclusão adotado foi trabalhar há, pelo menos, seis meses nas instituições e o de exclusão foi estar afastado de suas atividades, por qualquer motivo, durante a coleta de dados. Enquadraram-se nesses critérios 70 profissionais.

A coleta de dados ocorreu de novembro de 2017 a março de 2018 , por uma equipe formada por três estudantes do curso de enfermagem previamente treinados para a coleta. Abordaramse os prováveis participantes, em até três tentativas, e os profissionais que aceitaram participar do estudo foram encaminhados para salas reservadas nos próprios locais de trabalho, no qual, foram esclarecidos sobre o objetivo do estudo, assinaram o Termo de Consentimento Livre e Esclarecido (TCLE) e responderam o instrumento de coleta de dados que foi disponibilizado em envelope individual lacrado, para manter a confiabilidade e sigilo do participante.

Para a coleta de dados, utilizaram-se dois instrumentos, o primeiro de caracterização sociodemográfica e ocupacional, incluindo as seguintes variáveis: idade, sexo, estado civil, se 
possuía filhos, hábitos de vida: realiza exercício físico e nível de satisfação com as suas oportunidades de atividade de lazer e o ambiente de trabalho e ocupacionais: formação profissional, local de trabalho, turno, número de vínculos, jornada semanal, nível de satisfação no relacionamento com os colegas de trabalho e com o chefe imediato, satisfação com as oportunidades para expressar opiniões relacionadas ao trabalho, nível de equilíbrio entre sua vida profissional e pessoal/familiar, nível de conhecimento/habilidade para realizar as tarefas e renda individual mensal.

O segundo instrumento foi o Alcohol, Smoking and Substance Involvement Screening Test (ASSIST) que investiga o consumo de álcool e outras nove classes de drogas (tabaco, álcool, maconha, cocaína, anfetaminas, inalantes, hipnóticos/sedativos, alucinógenos, opioides e outras). Este é um instrumento validado e adaptado para a língua portuguesa apresentando bons níveis de confiabilidade, que recomendam sua utilização ${ }^{(9)}$. Esse instrumento inclui questões sobre a frequência de uso na vida; consumo nos últimos três meses; forte desejo/urgência em consumir nos últimos três meses; deixar de fazer alguma atividade nos últimos três meses, em decorrência do consumo da substância; problemas relacionados ao uso, tais como: saúde, financeiro, social, preocupação a respeito do consumo, por parte de pessoas próximas ao usuário; e tentativas de controlar/diminuir/cessar o uso. No entanto, para este estudo utilizaram-se, apenas, as questões de uso na vida, últimos três meses e forte desejo.

Para a análise dos dados, utilizou-se o programa Statistical Package for the Social Sciences (SPSS), versão 20.0. A estatística descritiva foi utilizada para descrever e resumir os dados obtidos. Foi empregada a análise univariada, por meio do teste qui-quadrado, para comparar a relação entre ambiente de trabalho com as características sociodemográficas, ocupacionais e o consumo de substâncias psicoativas dos profissionais da enfermagem.

Esta pesquisa foi submetida ao Comitê de Ética em Pesquisa da Universidade Estadual do Norte do Paraná, e obteve parecer favorável sob o no 2.211.294. Todos os participantes assinaram o TCLE e o estudo seguiu os pressupostos éticos definidos pela Resolução 466/2012.

\section{RESULTADOS E DISCUSSÃO}

Dos 70 profissionais de enfermagem, 35 atuavam na atenção básica e 35 nas instituições hospitalares. Ao comparar as características sociodemográficas e ocupacionais nota-se que sexo $(p=0,012)$, idade $(p=0,036)$, alimentação $(p=0,027)$ e formação $(p=0,002)$ foram estaticamente diferentes entre os grupos (Tabela 1).

Os profissionais de enfermagem, tanto no âmbito hospitalar quanto no âmbito na atenção básica, vivenciam cargas de trabalho com especificidades características de cada ambiente e rotina ocupacional. Frequentemente, essas especificidades vêm associadas ao excesso de demandas, condições precárias de trabalho, número insuficiente de recursos humanos e materiais, jornadas de trabalho excessivas, baixos salários, dentre outros fatores que interferem, negativamente, na assistência ao paciente $e$ provoca agravos a saúde desses profissionais ${ }^{(10)}$.

As cargas laborais provocam insatisfação, desgaste e adoecimento do trabalhador, além de dificultar a realização de um trabalho criativo e efetivo $^{(10)}$, o que pode contribuir para que o trabalhador utilize de estratégias para gerenciar esse sofrimento associado a sua prática laborativa, tais como: faltar ao trabalho, consumo abusivo de alimentos e a utilização de SPA ${ }^{(6,11)}$.

Enfatiza-se que, após a revolução industrial, o homem passou a buscar novas formas para maximizar o prazer e minimizar o sofrimento vivenciado em seu ambiente de trabalho, destacando-se o uso de SPA como uma estratégia rápida e eficaz para alcançar o bem-estar, aumentar o humor, socialização, relaxamento e diminuir o stress seja ele advindo do seu ambiente ocupacional ou relacionado à sua vida pessoal|(12).

No que concerne às características sociodemográficas dos participantes, verificou-se predominância do sexo feminino, adultos, casados, maioria técnicos de enfermagem e que atuavam no período diurno. Dados estes que vão de encontro com o cenário nacional da enfermagem, no qual a maioria dos profissionais são do sexo feminino $(84,6 \%)$ e da categoria de técnicos de enfermagem $(80 \%)^{(3,5,13)}$.

Vale ressaltar que, no mundo, a enfermagem possui aproximadamente 19,3 milhões de enfermeiros e, no Brasil, a categoria profissional representa metade da força de trabalho na área da saúde, representando um total de mais de dois milhões de profissionais, destes, $24 \%$ são enfermeiros, $56 \%$ técnicos de 
enfermagem e $20 \%$ auxiliar de enfermagem ${ }^{(14)}$, os quais estão inseridos em todos os níveis de atenção, desenvolvendo uma assistência direta ou indiretamente ao paciente.

No que concerne ao estado civil, verificouse que a maioria dos participantes do presente estudo eram casados. Estudos ${ }^{(3,6-7,11,15)}$ apontam que, indivíduos solteiros possuem padrões de consumo de álcool de maior risco ao comparar com os casados. Assim, a união estável se apresenta como um fator de proteção para o consumo de álcool, principalmente entre os homens, visto que, as mulheres, historicamente, possuem uma maior preocupação com sua saúde e procuram desenvolver hábitos de vida mais saudáveis ${ }^{(15)}$.

Outra caraterística de proteção é ter filhos, isto é, quando o profissional desenvolve um envolvimento afetivo com os filhos este se caracteriza como um fator que protege as pessoas para não consumir álcool, tabaco e outras drogas $^{(16)}$.

Ainda, ao analisar as características ocupacionais dos profissionais da enfermagem, no que diz respeito à satisfação com os colegas e com a chefia, os dados do presente estudo mostraram que há uma dualidade, ou seja, um percentual significativo de profissionais em ambos os locais laborais está satisfeito, mas ainda se percebe que ocorre a insatisfação. Percebe-se que, o cotidiano de trabalho da enfermagem, as condições de trabalho, dificuldades em lidar com o sofrimento, morte $e$ às relações interpessoais e interprofissionais conflituosas levam a uma maior insatisfação do profissional ${ }^{(17)}$. Todos esses fatores propiciam o aumento no consumo de drogas licitas e ilícitas pelos profissionais da enfermagem, visto que, o consumo nocivo/abusivo da SPA age como uma estratégia de defesa/fuga relacionado às demandas e insatisfações impostas no cotidiano laboral ${ }^{(18)}$.

Pesquisa desenvolvida com 1.608 trabalhadores da Noruega mostrou que, aqueles que possuem um risco aumento para o consumo de álcool apresentam um maior sofrimento psicológico e menor satisfação com o seu trabalho ao comparar-se com os trabalhadores que possuem um consumo nocivo de álcool(19).

Estudo $^{(20)}$ desenvolvido na China mostrou que, dificuldade nos relacionamentos interpessoais entre profissionais da saúde é algo vivenciado diariamente no ambiente de trabalho, sendo que, os principais fatores que influenciam o desenvolvimento de relações conflituosas entre esses profissionais tem destaque a má comunicação entre as equipes, o estresse excessivo e as prioridades de tarefas concorrentes. Todos esses fatores influenciam, negativamente, sobre a saúde do profissional, o que, por sua vez, favorece a redução na qualidade da assistência, aumenta os eventos adversos e, consequentemente, o esgotamento profissional, provocando o estresse ocupacional que propicia para o consumo de SPA seja ela na forma nociva ou abusiva ${ }^{(11,19,20-21)}$.

A maioria dos participantes do estudo em questão afirma não praticar exercício físico e ter uma alimentação inadequada. Estudo realizado com enfermeiros identificou que a prática de atividades físicas (moderada/alta ou de baixa intensidade) diminui em quase pela metade o risco para desenvolver problemas relacionados ao consumo de álcool quando comparado com o sedentarismo ${ }^{(23)}$.

Enfatiza-se que a prática de exercícios físicos e alimentação saudável são hábitos importantes para o desenvolvimento de uma boa qualidade de vida, quando o profissional possui características positivas quanto ao seu estilo de vida, consequentemente, esse hábito refletirá em um melhor rendimento na assistência, diminuição do estresse ocupacional e uma menor probabilidade para consumir SPA, visto que, o consumo de álcool se associa às dificuldades em gerenciar as demandas impostas pelo labor ${ }^{(3)}$.

Salienta-se que, a relação entre saúde e prática de atividade física é positiva, pois, essa prática atua na prevenção de doenças, assim como na manutenção e recuperação da saúde das pessoas em todas as faixas etárias. Um estilo de vida ativo, resultante da prática de atividade física, contribui para uma boa condição física, sendo este um fator importante para a prevenção e o tratamento de doenças, manutenção da saúde, bem como, um instrumento precioso para a melhoria da qualidade de vida e, consequentemente, um melhor desempenho em suas atividades laborais ${ }^{(9)}$.

Outro fator de risco para os profissionais da enfermagem é o desenvolvimento de Doenças Crônicas não Transmissíveis (DCNC), decorrentes dos fatores de riscos identificados como a falta de atividade física, alimentação inadequada, a obesidade e uso de álcool e outras drogas ${ }^{(1)}$. 
Tabela 1 - Características sociodemográficas e ocupacionais dos profissionais da enfermagem. Paraná, Brasil, 2020. $(n=70)$

\begin{tabular}{|c|c|c|c|c|c|}
\hline \multirow{2}{*}{ Variáveis } & \multicolumn{2}{|c|}{ Atenção básica } & \multicolumn{2}{|c|}{ Instituição hospitalar } & \multirow{2}{*}{ p-valor } \\
\hline & $n$ & $\%$ & $\mathbf{N}$ & $\%$ & \\
\hline \multicolumn{6}{|l|}{ Sexo } \\
\hline Masculino & 0 & 0 & 6 & 100 & \multirow{2}{*}{0,012} \\
\hline Feminino & 35 & 54,7 & 29 & 45,3 & \\
\hline \multicolumn{6}{|l|}{ Idade } \\
\hline 18 a 40 anos & 17 & 45,9 & 20 & 54,1 & \multirow{2}{*}{0,036} \\
\hline Acima de 40 anos & 15 & 53,6 & 13 & 46,4 & \\
\hline \multicolumn{6}{|l|}{ Estado civil } \\
\hline Casado & 21 & 56,8 & 16 & 43,2 & \multirow{2}{*}{0,169} \\
\hline Outro & 14 & 42,4 & 19 & 57,6 & \\
\hline \multicolumn{6}{|l|}{ Renda } \\
\hline Até 1 salário mínimo & 7 & 50 & 7 & 50 & \multirow{2}{*}{0,617} \\
\hline Mais de 1 salário mínimo & 28 & 50 & 28 & 50 & \\
\hline \multicolumn{6}{|l|}{ Alimentação } \\
\hline Ruim & 24 & 61,5 & 15 & 38,5 & \multirow{2}{*}{0,027} \\
\hline Boa & 11 & 35,5 & 20 & 64,5 & \\
\hline \multicolumn{6}{|l|}{ Exercício } \\
\hline Não & 24 & 53,3 & 21 & 46,7 & \multirow{2}{*}{0,309} \\
\hline Sim & 11 & 44 & 14 & 56 & \\
\hline \multicolumn{6}{|l|}{ Filhos } \\
\hline Não & 6 & 33,3 & 12 & 66,7 & \multirow{2}{*}{0,085} \\
\hline $\operatorname{Sim}$ & 29 & 55,8 & 23 & 44,2 & \\
\hline \multicolumn{6}{|l|}{ Formação } \\
\hline Nível técnico & 23 & 47,9 & 25 & 52,1 & \multirow{2}{*}{0,002} \\
\hline Graduação & 12 & 54,5 & 10 & 45,5 & \\
\hline \multicolumn{6}{|l|}{ Turno de Trabalho } \\
\hline Diurno & 35 & 72,9 & 13 & 27,1 & \multirow{2}{*}{0,232} \\
\hline Noturno & 0 & 0 & 22 & 100 & \\
\hline \multicolumn{6}{|l|}{ Satisfação com colega } \\
\hline Baixo & 13 & 50 & 13 & 50 & \multirow{2}{*}{0,598} \\
\hline Alta & 22 & 50 & 22 & 50 & \\
\hline \multicolumn{6}{|l|}{ Satisfação com chefe } \\
\hline Baixo & 12 & 42,9 & 16 & 57,1 & \multirow{2}{*}{0,232} \\
\hline Alta & 23 & 54,8 & 19 & 45,2 & \\
\hline
\end{tabular}

Fonte: Próprio autor.

Quando analisado o consumo de SPA (Tabela 2), entre os profissionais da enfermagem, identifica-se uma semelhança entre a população geral, sendo o álcool e o tabaco as substâncias mais consumidas, no entanto, o consumo de sedativos também foi evidenciado entre essa população. Estudos ${ }^{(3,6,11)}$ desenvolvidos com a mesma população também evidenciou as mesmas especificidades ao analisar o tipo de substância consumido na vida. Portanto, é possível afirmar que, as SPA encontram-se presentes nos mais diferentes grupos populacionais, não havendo distinção entre sexo, idade e escolaridade ${ }^{(22,24-25)}$.
Destaca-se o consumo do álcool como a substância mais consumida na vida $(68,6 \%)$, seguido por tabaco $(48,6 \%)$, maconha $(8,6 \%)$ e sedativo $(87,1 \%)$. Esses resultados se assemelham ao encontrado na literatura ${ }^{(3)}$, no qual, entre a população geral o álcool, tabaco e a maconha são as SPA mais consumidas em todo o mundo, configurando-se como um vultoso problema de saúde pública em nível mundial. Ainda, o uso de álcool e tabaco estão, diretamente, relacionados com o aumento da mortalidade prematura e anos de vida perdido por cargas de doenças relacionado à incapacidade funcional(2). 
Estudo desenvolvido com enfermeiros em Taiwan que teve como objetivo examinar os padrões de uso de benzodiazepínicos mostrou que, entre no período do estudo houve uma tendência crescente para a utilização entre esses profissionais, sendo que, enfermeiros com idade superior a 45 anos e aquelas com maior experiências, 5 anos ou mais, apresentou uma maior probabilidade para a utilização, bem como, entre aqueles que apresentam depressão, ansiedade e distúrbio do sono ${ }^{(23)}$. Assim, infere-se que, a utilização do sedativo observada, neste estudo, pode estar relacionada diretamente com as características ocupacionais vivenciadas pelos profissionais em seu ambiente de trabalho.

Tabela 2 - Características do consumo de substâncias psicoativas na vida, entre profissionais da enfermagem. Paraná, Brasil, 2020. $(n=70)$

\begin{tabular}{lllll}
\hline \hline \multirow{2}{*}{ Variável } & \multicolumn{2}{c}{ Sim } & $\mathbf{N}$ & Não \\
\cline { 2 - 5 } Uso na vida & $\mathbf{N}$ & & & \% \\
Tabaco & 34 & 48,6 & 36 & 51,4 \\
Álcool & 48 & 68,6 & 22 & 31,4 \\
Maconha & 6 & 8,6 & 64 & 91,4 \\
Cocaína & 4 & 5,7 & 66 & 94,3 \\
Anfetaminas & 3 & 4,3 & 67 & 95,7 \\
Inalantes & 3 & 4,3 & 67 & 95,7 \\
Hipnóticos/Sedativos & 9 & 12,9 & 61 & 87,1 \\
Alucinógenos & 3 & 4,3 & 67 & 95,7 \\
Opióides & 8 & 11,4 & 62 & 88,6 \\
Outros & 6 & 8,6 & 64 & 91,4 \\
\hline \hline
\end{tabular}

Fonte: Próprio autor.

No que concerne à comparação do uso de SPA entre os participantes do presente, na tabela 3, evidencia-se que o consumo de álcool e tabaco na vida e nos três últimos meses foi, proporcionalmente, maior entre os profissionais da atenção básica em relação à instituição hospitalar. Todavia, essa não foi uma diferença com significância estatística. Já o uso de sedativos foi maior entre aqueles de instituições hospitalares, sobretudo o uso na vida $(p=0,05)$. 0 forte desejo de consumo de álcool e de sedativos foi maior entre os profissionais das instituições hospitalares e para o tabaco foi entre os profissionais da atenção básica.

Tabela 3 - Comparação do consumo de álcool, tabaco e sedativos de acordo com o ambiente laboral. Paraná, Brasil, 2020. $(n=70)$

\begin{tabular}{|c|c|c|c|c|c|}
\hline \multirow{2}{*}{ Variáveis } & \multicolumn{2}{|c|}{ Atenção básica } & \multicolumn{2}{|c|}{ Instituição hospitalar } & \multirow{2}{*}{$\mathbf{P}$} \\
\hline & $\mathbf{N}$ & $\%$ & $\mathbf{N}$ & $\%$ & \\
\hline \multicolumn{6}{|c|}{ Uso na vida } \\
\hline Álcool & 27 & 56,2 & 21 & 43,8 & 0,19 \\
\hline Tabaco & 15 & 44,1 & 19 & 55,9 & 0,23 \\
\hline Sedativo & 4 & 44,4 & 5 & 55,6 & 0,05 \\
\hline \multicolumn{6}{|c|}{ Últimos 3 meses } \\
\hline Álcool & 18 & 58,1 & 13 & 41,9 & 0,16 \\
\hline Tabaco & 11 & 55,0 & 9 & 45,0 & 0,39 \\
\hline Sedativo & 1 & 25,0 & 3 & 75,0 & 0,30 \\
\hline \multicolumn{6}{|c|}{ Forte Desejo } \\
\hline Álcool & 7 & 46,7 & 8 & 53,3 & 0,52 \\
\hline Tabaco & 10 & 62,5 & 6 & 37,5 & 0,19 \\
\hline Sedativo & 0 & 0,0 & 1 & 100,0 & 0,48 \\
\hline
\end{tabular}

Fonte: Próprio autor. 
Estudo aponta que, fatores associados ao ambiente de trabalho da enfermagem como estresse, sobrecarga de trabalho, insatisfação, relacionamento interpessoal e déficit na comunicação pode contribuir para o aumento no consumo de sedativos, hipnóticos e antipsicóticos e, consequentemente, aumentar o risco de desenvolvimento de overdose ${ }^{(22)}$.

Corroborando com esses achados, estudo com enfermeiros de instituições hospitalares públicas constatou que o álcool, tabaco e sedativos foram as substâncias mais consumidas e que, quanto mais desfavorável for o ambiente de trabalho do enfermeiro, sobretudo na relação com médicos, suporte organizacional e autonomia, maior foi o consumo de SPA ${ }^{(11)}$. Assim, é notória a importância de proporcionar aos profissionais da enfermagem que atuam, tanto na atenção básica quanto na instituição hospitalar um ambiente favorável para sua prática profissional, visto que, essas características refletem, positivamente, para a sua saúde física e mental.

Acredita-se que os profissionais da enfermagem que não possuem hábitos e estilo de vida saudável estão mais propensos ao uso de SPA. Pesquisa realizada com profissionais da enfermagem de um hospital geral evidenciou que, comportamento de saúde inadequados/não saudáveis está diretamente relacionado para a prática de consumo nocivo/abusivo de álcool ${ }^{(3)}$.

A comparação entre os dois ambientes de trabalho investigado no presente estudo, constatou que ser profissional da enfermagem que atua no ambiente hospitalar apresentou uma maior frequência para forte desejo de consumo de álcool e sedativo. Sabe-se que, o processo laboral e as características ocupacionais entre os dois ambientes ocupacionais são distintas, porém, os problemas podem ser comuns como: a falta de materiais, a alta demanda de pacientes, falta de recursos humanos e materiais em quantidade e qualidade, dentre outros. Entretanto, as características inerentes às instituições hospitalares como horário de trabalho, complexidade do paciente, lidar com a morte, com a dor e insegurança/instabilidade no emprego são fatores que contribui para o abuso/dependência de álcool ${ }^{(7)}$.

Com relação aos profissionais da enfermagem que atuam na atenção básica, destaca-se que estes também estão expostos a riscos que podem favorecer o consumo de SPA. Esses profissionais vivenciam, diariamente, excesso de demanda, problemas estruturais e físicos e falha nas redes de atenção, o que dificulta a atuação profissional e que contribui para a insatisfação no trabalho e, por sua vez, contribui para uma maior possiblidade para o consumo de $\mathrm{SPA}^{(10)}$.

Ao analisar um estudo que abordou profissionais de enfermagem que atuam em equipes da Estratégia Saúde da Família, os resultados mostraram que $44,6 \%$ dos profissionais consumiam álcool no padrão binge, $16,2 \%$ apresentavam sintomas de depressão, 15,2\% de estresse e $23,2 \%$ de ansiedade, o qual, o uso de álcool em binge foi associado à depressão ( $p$ $=, 035$ ).

Assim, acredita-se que os profissionais da enfermagem estão adoecendo fisicamente e mentalmente, e utilizam as SPA como uma como estratégia de defesa, o qual pode estar associado com problemas pessoais relacionados com o seu ambiente extratrabalho, como também, problemas com o ambiente ocupacional, entre eles, a falta de autonomia, o suporte organizacional e o relacionamento médicoenfermeiro, fatores que contribuem para o consumo de SPA ${ }^{(12)}$.

Outro aspecto que pode estar associado ao consumo de sedativos, é a facilidade de acesso aos medicamentos controlados pela equipe de enfermagem. Essa é uma questão preocupante no contexto da enfermagem, pois o uso de substâncias psicotrópicas, assim como a depressão, pode estar relacionado às condições de trabalho, às dificuldades em lidar com o sofrimento e a morte, às relações interpessoais e interprofissionais e ao despreparo profissional. 0 consumo dessas substâncias objetiva diminuir as cargas físicas e psíquicas a que esses profissionais estão continuamente expostos ${ }^{(15)}$.

\section{CONCLUSÃO}

No presente estudo, foi possível observar que ocorre o uso de SPA entre os profissionais da enfermagem. Todavia, ao comparar as instituições estudadas, pode-se concluir que os sedativos e o álcool foram utilizados, predominantemente, por profissionais das instituições hospitalares, e o tabaco esteve mais presente entre os profissionais da atenção básica.

Embora o estudo tenha atingido o objetivo proposto, apresentou limitações em relação ao delineamento transversal que não possibilita o estabelecimento da relação causa e efeito, ao fato de ser uma pesquisa autorreferida, em que os entrevistados podem ter respondido o 
questionário, levando em consideração os padrões socialmente aceitáveis em seus ambientes. Dessa forma, sugere-se a realização de outros estudos.

Salienta-se que este estudo traz contribuições singulares, visto que a maioria das pesquisas brasileiras com os profissionais de enfermagem são desenvolvidas em cidades de médio e grande porte. Assim, ao mostrar a comparação do uso de SPA entre profissionais da enfermagem da atenção básica e de instituição hospitalar de uma cidade de pequeno porte há avanços, pois evidenciou uma realidade ainda pouco desvelada. Também, colabora para que os gestores em conjunto com os profissionais de enfermagem busquem ações que visem a eliminar o consumo dessas drogas e por sua vez promover a saúde.

\section{REFERÊNCIAS}

1- Bardach AE, Alcaras AO, Ciapponi A, Garay OU, Riviere AP, Palacios A, et al. Alcohol consumption's attributable disease burden and costeffectiveness of targeted public health interventions: A systematic review of mathematical models. BMC Public Health 2019;19:1378. DOI: 10.1186/s12889-019-7771-4

2- Atzendorf J, Rauschert C, Seitz NN, Lochbühler $\mathrm{K}$, Kraus $\mathrm{L}$. The use of alcohol, tobacco, illegal drugs and medicines. Dtsch Arztebl Int. 2019;116(35/36):577-84. DOI: 10.3238/arztebl.2019.0577

3- Junqueira MAB, Ferreira MCM, Soares GT, Brito $I E$, Pires PLS, Santos MA, et al. Uso de álcool e comportamento de saúde entre profissionais da enfermagem. Rev Esc Enferm USP 2017;51:1-8. DOI: $10.1590 / \mathrm{s} 1980-220 \times 2016046103265$

4- Jomar RT, Abreu ÂMM, Griep RH. Padrões de consumo de álcool e fatores associados entre adultos usuários de serviço de atenção básica do Rio de Janeiro, RJ, Brasil. Ciênc Saúde Coletiva 2014;19(1):27-38. DOI: $10.1590 / 1413-$ $\underline{81232014191.2009}$

5- Thompson A, Choque JCH, Jorgenson E, Yin J, Kinnunen T. Functional validity, role, and implications of heavy alcohol consumption genetic loci. Sci Adv. 2020;6(3):eaay5034. DOI: 10.1126/sciadv.aay5034

6- Scholze AR, Martins JT, Grandi AL, Galdino MJQ, Robazzi MLCC. Uso de substâncias psicoativas entre trabalhadores da enfermagem. Rev Port Enferm Saúde Mental 2017;18:23-30. DOI: $\underline{10.19131 / \text { rpesm.0188 }}$
7- Diniz CFG, Assunção AÁ, Beinner MA, Pimenta AM. Abuso/dependência de álcool e fatores psicossociais do trabalho em profissionais de saúde. Ciênc Cuid Saúde 2019;18(3):1-9. DOI: 10.4025/cienccuidsaude.v18i3.45023

8- Januário GC, Carvalho PCF, Lemos GC, Gir E, Toffano SEM. Acidentes ocupacionais com material potencialmente contaminado envolvendo trabalhadores de enfermagem. Cogitare Enferm. 2017;22(1):1-9. DOI: 10.5380/ce.v22i1.48893

9- Henrique IFS, De Micheli D, Lacerda RB, Lacerda LA, Formigoni MLOS. Validação da versão brasileira do teste de triagem do envolvimento com álcool, cigarro e outras substâncias (ASSIST). Rev Assoc Med Bras. 2004;50(2):199-206. DOI: 10.1590/S0104-4230200400020003

10- Pires DEP, Machado RR, Soratto J, Scherer MA, Gonçalves ASR, Trindade LL. Cargas de trabalho da enfermagem na saúde da família: Implicações no acesso universal. Rev Latino-Am Enfermagem 2016;24:1-9. DOI: 10.1590/1518$\underline{8345.0992 .2682}$

11- Scholze AR, Martins JT, Galdino MJQ, Ribeiro RP. Ambiente ocupacional e o consumo de substâncias psicoativas entre enfermeiros. Acta Paul Enferm. 2017;30(4):404-11. DOI: 10.1590/1518-8345.0992.2682

12- Taggar JS, Lewis S, Docherty G, Bauld L, McEwen A, Coleman T. Do cravings predict smoking cessation in smokers calling a national quit line: Secondary analyses from a randomised trial for the utility of "urges to smoke" measures. Subst Abuse Treat Prev Policy 2015;10:1-10. DOI: 10.1186/s13011-015-0011-8

13- Conselho Federal de Enfermagem. Pesquisa inédita traça o perfil da enfermagem brasileira Brasília: COFEN; 2015. Disponível em: http://www.cofen.gov.br/pesquisa-inedita-tracaperfil-da-enfermagem 31258.html

14- Biff D, Pires DEP, Forte ECN, Trindade LL, Machado RR, Amadigi FR, et al. Cargas de trabalho de enfermeiros: Luzes e sombras na Estratégia Saúde da Família. Ciênc Saúde Coletiva 2020;25(1):147-58. DOI: 10.1590/141381232020251.28622019

15- Bertussi VC, Junqueira MABB, Giuliani CD, Calçado RM, Miranda FJS, Santos MA, et al. Substâncias psicoativas e saúde mental em profissionais de enfermagem da Estratégia Saúde da Família. Rev Eletr Enf. 2018;20/21:1-9. DOI: 10.5216/ree.v20.47820

16- Ferro L, Meneses-Gaya C. Resiliência como fator protetor no consumo de drogas entre 
universitários. Saude e Pesqui. 2015 [citado em 7 fev 2020]; 8(nesp):139-49. Disponível em: https://periodicos.unicesumar.edu.br/index.php/ saudpesq/article/view/3774/2519

17- Almazan JU, Albougami AS, Alamri MS. Exploring nurses' work-related stress in an acute care hospital in KSA. J Taibah Univ Med Sci. 2019;14(4):376-82.

DOI:

10.1016/i.jtumed.2019.04.006

18- Vieira GCG, De Brida RL, Macuch RS, Massuda EM, Preza GP. Uso de psicotropicos pelo enfermeiro: Sua relação com o trabalho. Cinergis 2016;17(3):191-5.

DOI: 10.17058/cinergis.v17i3.8118

19- Nielsen MB, Gjerstad J, Frone M. Alcohol use among Norwegian workers: Associations with health and well-being. Occup Med. 2018;68(2):9698. DOI: $10.1093 /$ occmed/kqy014

20- Jerng JS, Huang SF, Liang HW, Chen LC, Lin CK, Huang HF, et al. Workplace interpersonal conflicts among the healthcare workers: Retrospective exploration from the institutional incident reporting system of a university-affiliated medical center. PLoS One 2017;12(2):e0171696. DOI: 10.1371/journal.pone.0171696

21- Lin CF, Huang $\mathrm{Cl}$, Yang $\mathrm{CM}$, Lu MS. The relationship between work environment satisfaction and retention intention among nursing administrators in Taiwan. J Nurs Res. 2019;27(5):e43.

DOI: 10.1097/jinr.0000000000000312

22- Ke Y-T, Feng IJ, Hsu C-C, Wang J-J, Su S-B, Huang $\mathrm{C}-\mathrm{C}$, et al. Nurses have a four-fold risk for overdose of sedatives, hypnotics, and antipsychotics than other healthcare providers in Taiwan. PLoS One 2018; 13(8):e0202004. DOI: 10.1371/journal.pone.0202004

23- Sang E, Liao YM, Miao NF, Chou KR, Chung $\mathrm{MH}$. Patterns and correlates of benzodiazepine use in nurses: A nationwide, population-based study. Int J Ment Health Nurs. 2018;27(1):400-7. DOI: 10.1111/inm.12334

24- Perry L, Xu X, Gallagher R, Nicholls R, Sibbritt $D$, Duffield C. Lifestyle health behaviors of nurses and midwives: The 'Fit for the Future' Study. Int J Environ Res Public Health 2018;15(5):945. DOI: 10.3390/ijerph15050945

25- Abreu AMM, Parreira PMSD, Souza MHN, Barroso TMMDA. Perfil do consumo de substâncias psicoativas e sua relação com as características sociodemográficas: Uma contribuição para intervenção breve na atenção primária à saúde, Rio de Janeiro, Brasil. Texto
Contexto Enferm. 2016;25(4):1-9. DOI: 10.1590/0104-07072016001450015

Nota: Este artigo integra a monografia de final de curso "Uso de substâncias psicoativas entre profissionais da enfermagem da atenção primária a saúde e hospitalar" do curso de enfermagem da Universidade Estadual do Norte do Paraná (UENP).

Recebido em: 16/04/2020

Aprovado em: 05/08/2020

Endereço de email:

Alessandro Rolim Scholze

Rua Prefeito José Mario Junqueira, 393 ap. 01, CEP: 86.360000, Bandeirantes (PR).

E-mail: scholze@uenp.edu.br 